\title{
Publisher's Note: Negative pressure in shear thickening band of a dilatant fluid
} [Phys. Rev. E 94, 062614 (2016)]

Shin-ichiro Nagahiro and Hiizu Nakanishi

Q (Received 6 March 2018; published 12 March 2018)

DOI: 10.1103/PhysRevE.97.039901

This paper was published online on 29 December 2016 with errors in the caption to Fig. 3. The fourth sentence of caption to Fig. 3 should read as "(c), (d) Normal pressures for $\mathrm{S}_{e}=1.05 \mathrm{kPa}$ and for $\mathrm{S}_{e}=0.84 \mathrm{kPa}$." The caption has been corrected as of 6 March 2018. The caption is incorrect in the printed version of the journal. 